Study design This was a qualitative observational study with analysis of pre and post interventional audit results.

Intervention and Strategy for Change Multidisciplinary communication with various stakeholders was key in agreeing common goals to improve efficiency and quality of the current theatre list. Using a Plan-do-Study-Act Cycle changes were gradually introduced over the next 4 months. The teams were updated by regular weekly emails and monthly meetings to discuss the next steps.

Staff Resources: Rota allocation to ensure optimal junior medical staff cover on the procedure days to allow prompt start. Improved training of doctors and allocating two doctors to a procedure list, one of whom is an experienced senior doctor who can provide immediate assistance to the junior colleague. In addition an Advanced Nurse Practioner has been appointed to start a nurse led service. Improved communication with anaesthetic collegues and availability of their contact details.

Time management: Inter-patient delays were approached by improved co-ordination amongst the recovery and day care nurses and patient transport strategies.

Booking system: With increasing patient numbers it was essential to have an improved electronic booking system. An appointment scheduler giving staggered appointment times would be introduced. Pending this, patients were encouraged to arrive before 10 am and have necessary investigations done in time prior to their procedure.

Sample delivery: Introduction of specific porters for timely collection of samples enabling quick diagnosis of new oncology children. Re-organisation of the procedure order so that diagnostic bone marrows are first on the list.

Measurement and effects of change Descriptive statistics was used and nominal variables calculated as percentages. The results are:-

1. $85 \%$ reduction in late finish and cancelled procedures

2. $100 \%$ of the samples delivered to laboratory by $5 \mathrm{pm}$

3. $80 \%$ improvement in availability of pre-procedure blood results

4. Improved training of doctors and team morale

Lessons learnt Inclusion of patient satisfaction surveys would have enabled us to include their views and make modifications to the service.

Message for others Good planning with adherence to SMART objectives is essential

Early involvement of stake holders and agreement on common goals

To ensure sustainability we involved the permanent day care nurses and senior managers.

\section{G542(P) PLANNING AHEAD: IMPLEMENTATION OF RESPIRATORY SUPPORT FOR BRONCHIOLITIS AT DESIGNATED WARD AREAS OUTSIDE PICU BEFORE THE SEASON}

B Tharayil, D Jyothish. General Paediatrics, Birmingham Children's Hospital, Birmingham, UK

\subsection{6/archdischild-2015-308599.493}

Context The General Paediatric department in the major children's teaching hospital is a large and busy unit with Paediatric intensive care unit (PICU) on-site. There are approximately 4300 admissions per annum. In-patient numbers vary considerably between the seasons with an average range of $80 \pm 10$ in the winter.

Problem A significant number of winter admissions where infants with bronchiolitis and a proportion require respiratory support with high flow nasal cannula oxygen therapy (HFNC).

Assessment of problem and analysis of its causes With this in mind we analysed the existing bronchiolitis patient pathway. We identified HFNC is only provided in PICU due to staff competencies. This has lead to loss of PICU bed resulting in cancellation of elective cardiac surgeries and refusal of retrievals.

Intervention A focus group was formed following the analysis to look at the feasibility of HFNC outside PICU. A strategy with a combined focus on safety and effective treatment with HFNC for children with bronchiolitis, nursed in designated Medical High Dependency Unit (MHDU) beds was developed.

Study design The 'Plan Do Study Act'(PDSA) cycle guided iterative stages of the HFNC project development. The normalisation process theory helped to introduce the new intervention providing the right treatment for the right patient at the right time without delay.

Strategy for change The equipment designed specifically for delivering humidified nasal high flow with in-built safety alarm was identified and it was procured for the HFNC project. The designated MHDU beds were identified on the same floor at one place rather than spreading it out across. We identified the optimum and relevant staffs; nurses, doctors and advance nurse practitioners (ANP); to be trained after estimating the capacity and demand for services to manage the new change safely. More than $90 \%$ of medical team was trained and all nurses in designated areas were also trained over the period with multiple dedicated sessions by three trainers supported by the representative from the company. A hospital grand round presentation was done before starting the project. An intranet hospital link was developed with training materials and standard operating procedure (SOP). A DVD guide showing all aspects of HFNC was also developed and included in the link.

Measurement of improvement We are currently in the stage of seeing the positive effects of HFNC project and data is being collected prospectively.

Effects of changes A collaborative approach was undertaken for the improvement of HFNC for bronchiolitis outside PICU. The process involved clinicians, nurses and managers in the redesign of bronchiolitis treatment pathway. By maintaining and adhering to a standardised operating document, a culture of continuous re-inforcement and support with clear guideline and consistency helped in the implementation of this new change and effective treatment successfully in the bronchiolitis patient pathway.

Lessons learnt There were barriers like competing priority of the professionals involved to achieve the same goal, clarity of role in delivering change and accountability and training of relatively large number the staff.

Message for others It is important to ensure stakeholder engagement from the outset at the start. Having trust, rapport and confidence with the wider team have hugely helped for the acceptance without any hindrance. It is very crucial to recognise the potential pitfalls and develop preventative actions accordingly. The task for this project was not to fixate on the cost side of the equation - saving money or making cuts - but to improve the productivity. The focus was on the benefits side of providing the right treatment for the right patient at designated place safely thereby increasing the value of care to patients. 